\title{
Duodenal perforation as a consequence of biliary stent migration can occur regardless of stent type or duration
}
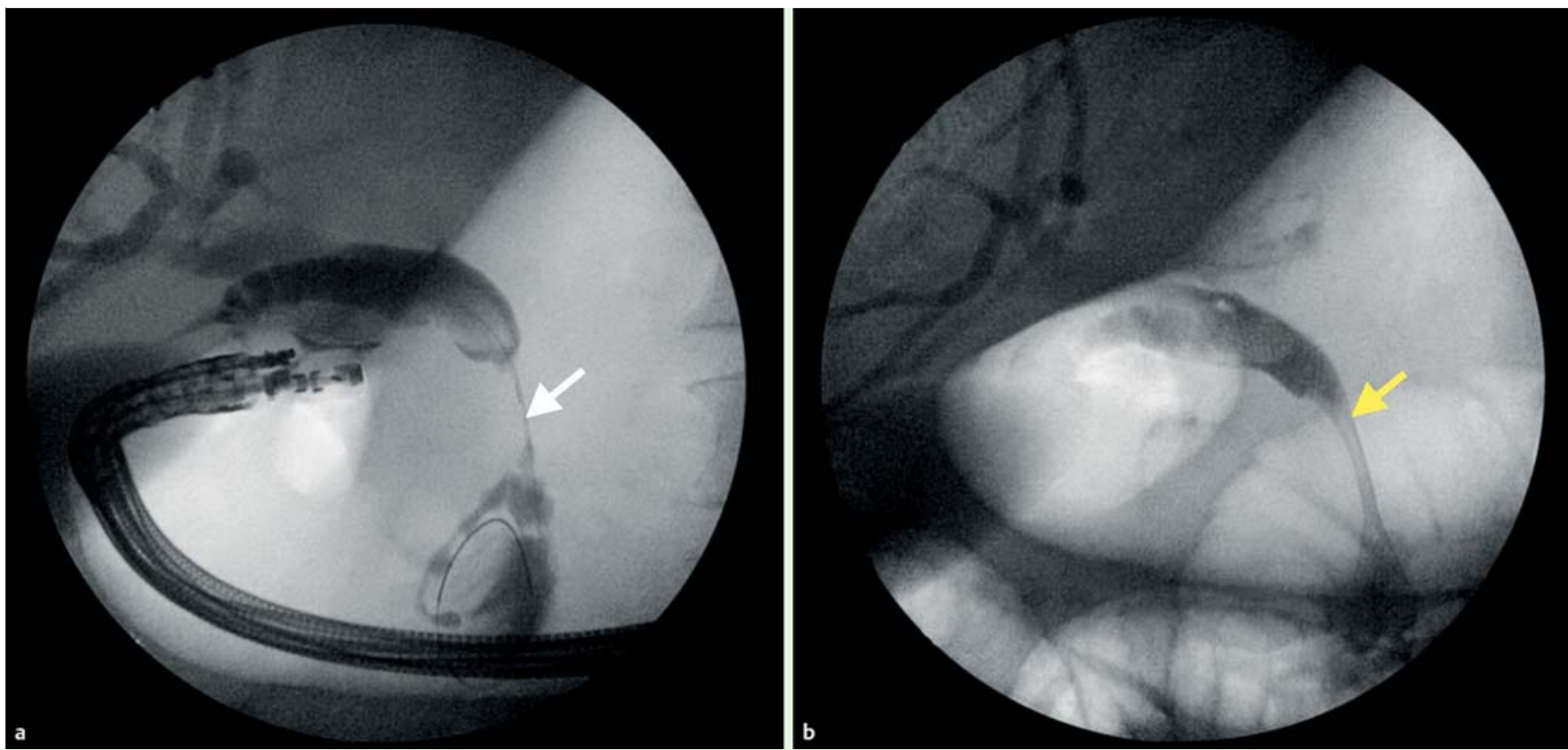

Fig. 1 Fluoroscopic images schowing: a succesful wire insertin through the bile duct into the duodenum, crossing a 3-cm stricture (white arrow), b a metalbiliary stent being depolyed across the biliary stricture (yellow arrow).

Biliary stents are the preferred method of managing malignant and benign biliary obstruction [1]. However, they are not without potential complications, including occlusion, migration, and intestinal perforation [1]. We report two cases of duodenal perforation as a result of migration of metal and plastic biliary stents.

A 70-year-old man with biliary obstruction secondary to an adenocarcinoma of the pancreatic head and gastric outlet obstruction that prevented transpapillary biliary access underwent antegrade placement of a 6 -cm fully covered metal biliary stent (Wallflex $10 \mathrm{~mm} \times 60 \mathrm{~mm}$; Boston Scientific, Natick, Massachusetts, USA) using an endoscopic ultrasound (EUS)guided antegrade technique ( $\bullet$ Fig. 1 ).

He presented 3 months later with diabetic ketoacidosis. An abdominal computed tomography (CT) scan revealed evidence of a mild pneumoperitoneum ( Fig.2) An exploratory laparotomy identified an intraperitoneal perforation of the third portion of the duodenum. The stent was removed, gastrointestinal flow was diverted with an antrectomy and gastrojejunostomy, and a percutaneous biliary tube was inserted for biliary diversion.

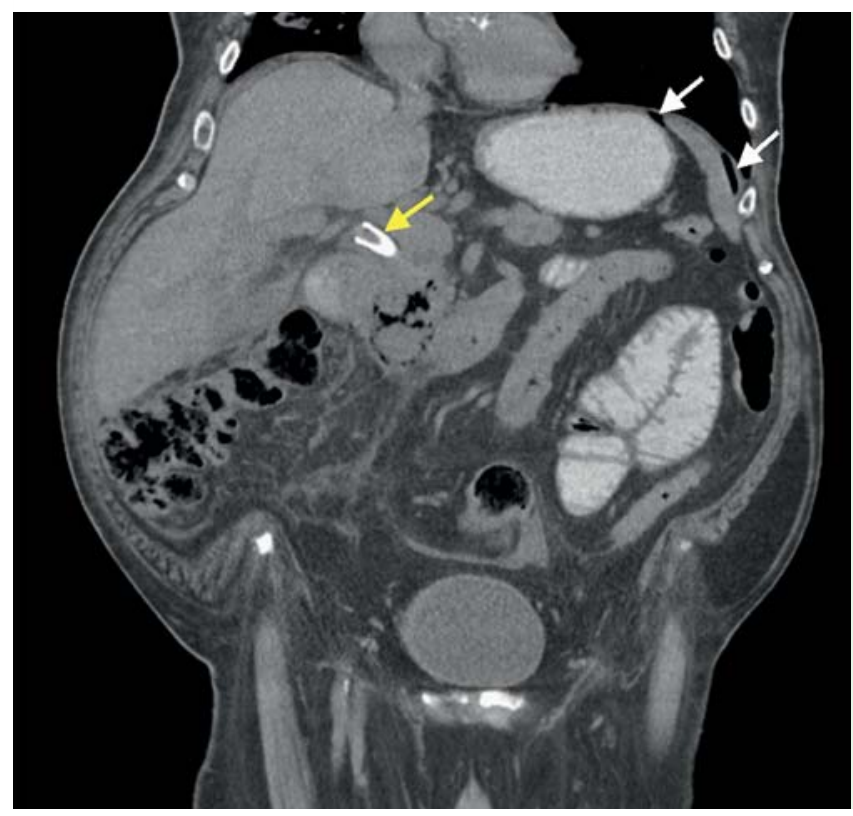

Fig. 2 Coronal computed tomography (CT) scan of the abdomen showing the metal stent (yellow arrow) and the pneumoperitoneum (white arrows).

Following this, the patient recovered and he was discharged after 7 days.

A 67-year-old man underwent endoscopic retrograde cholangiopancreatography (ERCP) with biliary sphincterotomy and insertion of a $10-\mathrm{Fr} \times 12-\mathrm{cm}$ straight plastic biliary stent (Cotton-Leung; Wil-
son-Cook, Winston-Salem, North Carolina, USA) for management of an anastomotic biliary stricture ( $\bullet$ Fig.3) after an orthotopic liver transplant.

The patient presented 1 week after the ERCP with fevers and elevated values on liver function testing. A CT scan revealed 


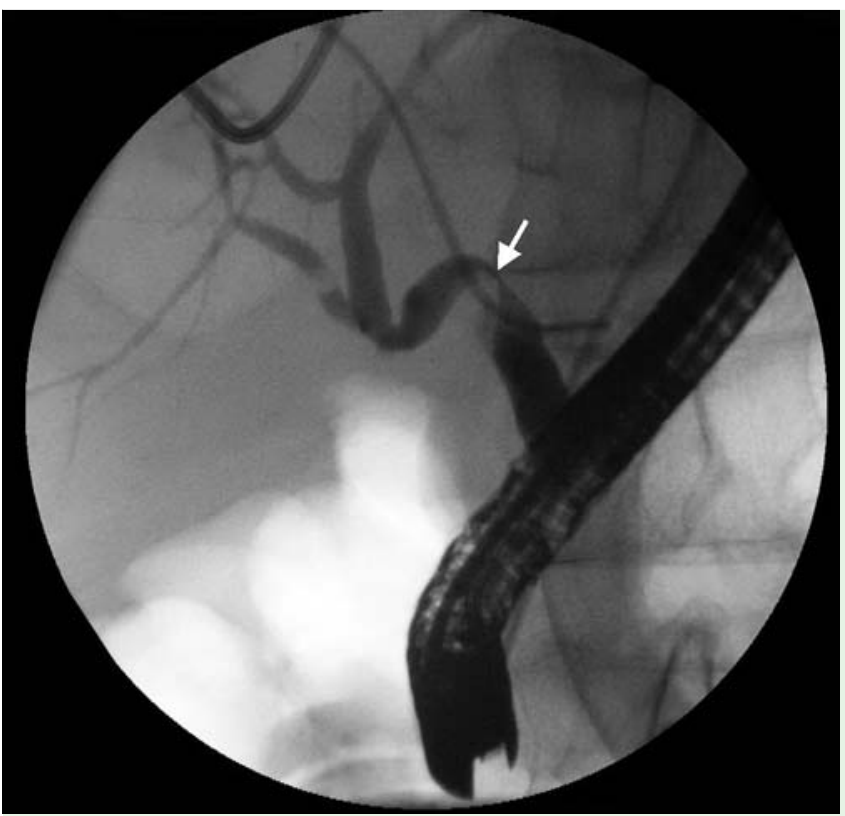

Fig. 3 Cholangiogram in a second patient showing an anastomotic stricture (white arrow) that required placement of a $12-\mathrm{cm}$ plastic stent.

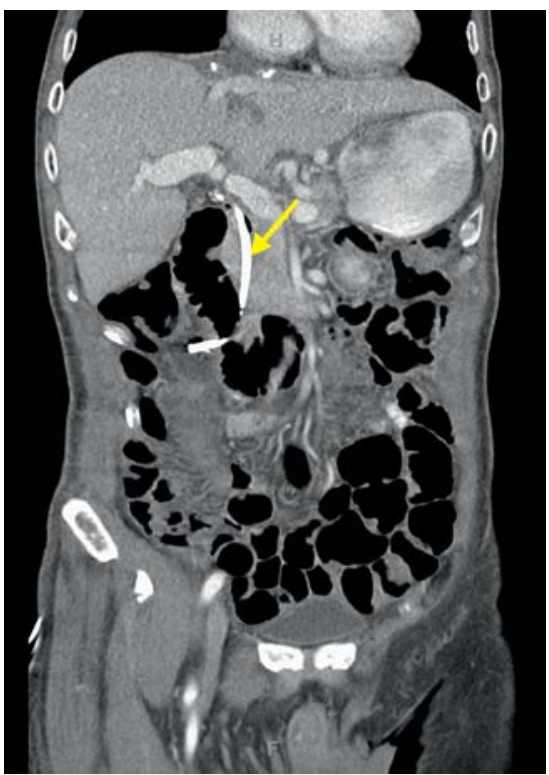

Fig. 4 Coronal computed tomography (CT) scan of the abdomen showing that the plastic stent (yellow arrow) had migrated and perforated through the duodenal wall.

signs of a sealed perforation in the second portion of the duodenum ( Fig.4). An intraoperative ERCP was performed ( $\bullet$ Fig.5), followed by an exploratory laparotomy with repair of the duodenal perforation with a Graham patch and a jejunal serosal patch. A percutaneous biliary drain was inserted. The patient died 4 weeks later because of sepsis and multiorgan failure.

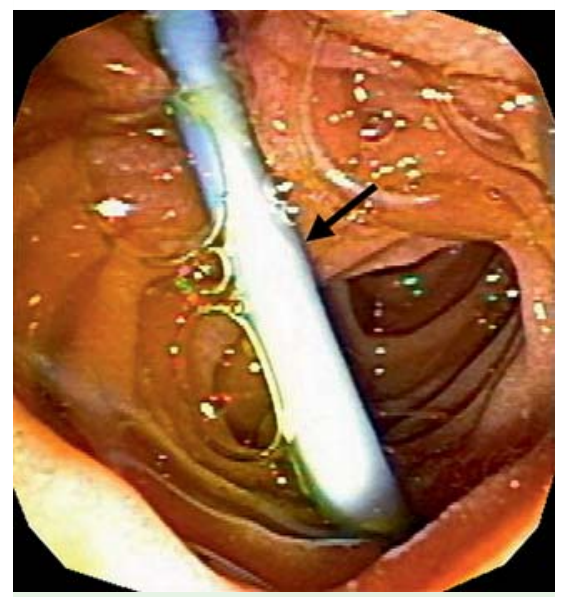

Fig. 5 Endoscopic image during an intraoperative endoscopic retrograde cholangiopancreatography (ERCP) showing the plastic stent that had migrated out of the biliary tree through the duodenal wall (black arrow).

Migration of biliary stents, whether metal or plastic, has been reported to occur in $8 \%-10 \%$ of cases; however, subsequent perforation is a rare event [2]. Notably, patients can present with nonspecific symptoms; therefore, a high index of suspicion for perforation should be maintained when evaluating these patients, irrespective of the duration of the stent placement $[3,4]$. Our two cases demonstrate that duodenal perforation can occur in both benign and malignant settings, regardless of the type of stent or its length of time in place.

\section{Endoscopy_UCTN_Code_CPL_1AL_2AD}

Competing interests: Mouen A. Khashab is a consultant for Boston Scientific and Olympus America and has received research support from Cook Medical. Payal Saxena has received consultancy fees from Boston Scientific and has received research support from Cook Medical.

\section{Mohamad H. El Zein', Vivek Kumb- hari $^{1}$, Alan Tieu ${ }^{1}$, Payal Saxena ${ }^{1}$, Ahmed A. Messallam¹, Alba Azola', Zhiping Li' ${ }^{1}$, Matthew Weiss ${ }^{2}$, Mouen A. Khashab ${ }^{1}$}

${ }^{1}$ Department of Medicine and Division of Gastroenterology and Hepatology, The Johns Hopkins Medical Institutions, Baltimore, Maryland, USA

2 Department of Surgery, The Johns Hopkins Medical Institutions, Baltimore, Maryland, USA

\section{References}

1 Issa $H$, Nahawi M, Bseiso B et al. Migration of a biliary stent causing duodenal perforation and biliary peritonitis. World J Gastrointest Endosc 2013; 5: 523-526

2 Cerisoli C, Diez J, Gimenez M et al. Implantation of migrated biliary stents in the digestive tract. HPB (Oxford) 2003; 5: 180-182

3 Bharathi RS, Rao PP, Ghosh K. Intra-peritoneal duodenal perforation caused by delayed migration of endobiliary stent: A case report. Int J Surg 2008; 6: 478-480

4 Miller G, Yim D, Macari M et al. Retroperitoneal perforation of the duodenum from biliary stent erosion. Curr Surg 2005; 62: 512 515

\section{Bibliography}

DOI http://dx.doi.org/

10.1055/s-0034-1365790

Endoscopy 2014; 46: E281-E282

(c) Georg Thieme Verlag KG

Stuttgart · New York

ISSN 0013-726X

\section{Corresponding author}

\section{Mouen A. Khashab, MD}

Division of Gastroenterology and Hepatology

Johns Hopkins Hospital

1800 Orleans St, Suite 7125 B

Baltimore

MD 21205

USA

Fax: +1-443-683-8335

mkhasha1@jhmi.edu 